\title{
Noise and Dynamic Range of CMOS Degenerated Active Inductor Resonators
}

\author{
Grzegorz Szczepkowski*, Ronan Farrell ${ }^{\dagger}$ \\ Center for Telecommunications Value Chain Research \\ National University of Ireland Maynooth \\ Email: *gszczepkowski@eeng.nuim.ie, ${ }^{\dagger}$ rfarrell@eeng.nuim.ie
}

\begin{abstract}
This paper presents a compensation method of active inductor losses, employing passive gyrator degeneration rather than an external negative impedance converter. Theoretical analysis is confirmed by a comparative simulations of two resonators designed for $434 \mathrm{MHz}$ band, using Eldo RF and Spectre and the UMC 0.18 $\mu \mathrm{m} \mathrm{1P6M}$ process. Presented results show that the proposed loss compensation method achieves comparable noise and large signal performance to a standard active inductor resonator and provides a significant improvement in static power consumption.
\end{abstract}

\section{INTRODUCTION}

Inductors synthesized using active devices offer a number of advantages over their spiral counterparts in terms of chip area and tuning ability. This has led to continued interest for use in filters and oscillators, for high frequency wireless and wired communications and other niche applications. However, practical implementations remain few as active inductors suffer from poor noise performance, limited dynamic range and high power consumption. An additional restraint is the lack of robust design guidelines, though the first gyrator-based active inductor was presented by Tellegen in 1948 [1] In this paper, a new design methodology for the degenerated active inductor will be presented that allows a tradeoff between noise, power and dynamic range. Supplementing this methodology is a new noise analysis for the impact of degeneration in an active inductor.

\section{GROUNDED ACTIVE INDUCTOR RESONATOR}

The fundamental structure of a gyrator-based active inductor, as presented in Fig. 1. [1], consists of two generic transconductance amplifiers in back to back connection and loaded with a single capacitor.

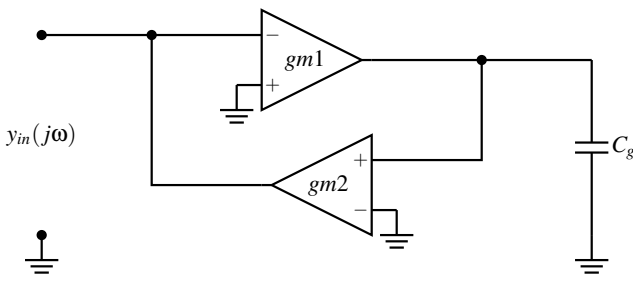

Fig. 1. Ideal grounded active inductor.

The input small signal admittance seen on the unloaded port is equal to

$$
y_{\text {in }}(j \omega)=\frac{1}{j \omega L_{\text {pai }}}
$$

where

$$
L_{p a i}=\frac{C_{g}}{g m_{1} g m_{2}}
$$

To compensate for the low $\mathrm{Q}$ values of the simple resonator, a negative resistor is used. Typical solutions include a single transistor grounded negative resistor or a cross-coupled differential pair, shown in Fig. 2. The latter circuit is more suitable for integration as it consumes less power and does not require external components to generate negative resistance [2]. If both transistors have large DC gain, the differential input admittance of the circuit is

$$
y_{\text {indiff }_{\text {dif }}}(j \omega)=G_{a c t_{\text {diff }}}+j \omega C_{a c t_{\text {diff }}}=\frac{-g m_{e x}}{2}+j \omega \frac{C_{g s}}{2}
$$

The static power necessary to achieve negative resistance is proportional to the resonator losses as well as the capacitance value of the tank [3].

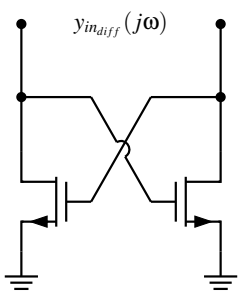

Fig. 2. NMOS cross-coupled negative resistor

\section{DEgENERATED ACTIVE INDUCTOR RESONATOR}

This section describes a method of compensating the active inductor losses by utilizing the concept of gyrator degeneration [4]. Wang and Abidi [5] showed that parasitic effects of transconductor capacitances and conductances create undesirable negative resistive component at the gyrator input, causing peaking of the resonator transfer function or even instability. However, the same effect can be used for compensation of the active inductor losses, avoiding the need for an external Q-enhancing circuit and thus minimizing DC power consumption. This is especially useful for gyrator circuits where losses can be large.

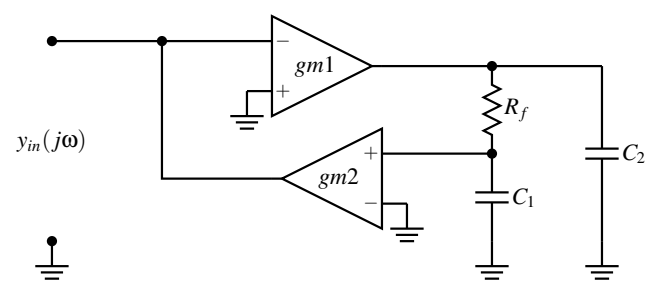

Fig. 3. Degenerated active inductor with negative input conductance.

Fig. 3. presents the basic concept of degenerated active inductor Degeneration is obtained by introducing an $\mathrm{RC}$ circuit at the output of the first transconductor. The capacitor voltage $\left(C_{1}\right)$ is then used to drive a feedback amplifier. As a result, the input admittance of the circuit consists of a negative parallel conductance and an inductance

$$
y_{i n}(j \omega)=G_{d g}+\frac{1}{j \omega L_{p d g}}
$$


By introducing variables

$$
\begin{aligned}
C_{p} & =C_{1}+C_{2} \\
C_{s} & =\frac{C_{1} C_{2}}{C_{1}+C_{2}}
\end{aligned}
$$

and

$$
\omega_{H}=\frac{1}{C_{S} R_{f}}
$$

the components of (4) are equal to

$$
\begin{aligned}
G_{d g} & =\frac{-g m_{1} g m_{2}}{\omega_{H} C_{p}\left[1+\left(\frac{\omega}{\omega_{H}}\right)^{2}\right]} \\
L_{p d g} & =\frac{C_{p}}{g m_{1} g m_{2}}\left[1+\left(\frac{\omega}{\omega_{H}}\right)^{2}\right]
\end{aligned}
$$

and

$$
G_{d g}=\frac{-1}{\omega_{H} L_{p d g}}
$$

which corresponds to the generic derivation presented by Wang and Abidi [5]. By introducing resistor $R_{f}$ the overall noise of the degenerated gyrator will increase. However, the total resonator noise may still be comparable to the one using a traditional active inductor tank.

\section{NOISE ANALYSIS}

For a comparison of total noise generated in a standard and degenerated resonator circuits, both tanks have to be equal in terms of following parameters: resonant frequency, tank capacitance $C_{t}$, active inductor power consumption and overall losses $G_{t}$. In addition, to maximise the dynamic range of an active inductor resonator, capacitances $C_{g}$ and $C_{t}$ should be equal, as advised by Cranickx [3] Therefore, at the resonant frequency

$$
\begin{gathered}
L_{p a i} \equiv L_{p d g} \\
G_{t} \equiv\left|-G_{a c t}\right| \equiv\left|-G_{d g}\right|
\end{gathered}
$$

which depending on $R_{f}$ yields numerous valid combinations of $C_{1}$ and $C_{2}$ calculated from (7). To start, the total output noise of the traditional tank is derived. Fig. 4 presents noise model of the compensated active inductor resonator. A parallel conductance $G_{l}$ represents the load which controls the overall quality factor of the tank. The output noise is represented as an output voltage of the resonator.

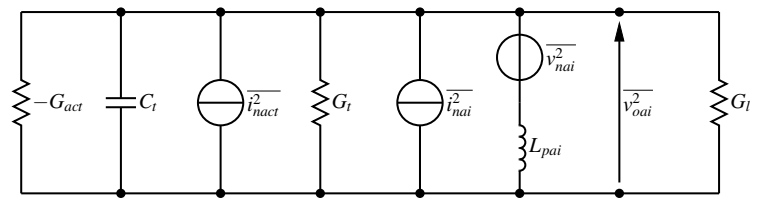

Fig. 4. Noise model of an active inductor resonator with negative resistor.

The total noise power of any circuit, $\overline{v_{\text {ntot }}^{2}}$ or $\overline{i_{\text {ntot }}^{2}}$, assuming uncorrelated noise sources, is given as

$$
\overline{v_{n t o t}^{2}}, \overline{i_{n t o t}^{2}}=\sum_{k=1}^{N}\left(\frac{\overline{v_{k}^{2}}}{\Delta f}, \frac{\overline{i_{k}^{2}}}{\Delta f}\right) \int_{0}^{\infty}\left|T_{k}(j \omega)\right|^{2} d f
$$

For frequencies relatively close to resonance, the negative resistor compensates all tank losses, leaving only the load conductance $G_{l}[6]$
The noise transfer function for current generators is thus equal to

$$
Z_{\text {ntai }}(j \omega)=\frac{j \omega L_{\text {pai }}}{j \omega L_{\text {pai }} G_{l}+1-\omega^{2} C_{t} L_{\text {pai }}}
$$

and similarly for active inductor voltage noise generators

$$
A_{n t a i}(j \omega)=\frac{1}{j \omega L_{p a i} G_{l}+1-\omega^{2} C_{t} L_{p a i}}
$$

Assuming that each transconductor is represented by a single transistor and for simplicity of calculations only thermal channel noise is considered, active inductor noise sources are equal to [7]

$$
\begin{aligned}
& \frac{\overline{i_{n a i}^{2}}}{\Delta f}=\frac{\overline{i_{d 2}^{2}}}{\Delta f}=4 k T \gamma g m_{2} \\
& \frac{\overline{v_{\text {nai }}^{2}}}{\Delta f}=\frac{1}{g m_{1}^{2}} \frac{\overline{i_{d 1}^{2}}}{\Delta f}=\frac{4 k T \gamma}{g m_{1}}
\end{aligned}
$$

where $\gamma$ is the MOS noise coefficient (technology dependent, varies between 2 and 5 for a short channel transistors) [2]. For cross-coupled negative resistor consisting of two equal noise current sources

$$
\overline{\frac{i_{\text {nact } t_{1}}^{2}}{\Delta f}}=\frac{\overline{i_{\text {nact }}^{2}}}{\Delta f}=4 k T \gamma g m_{e x}
$$

Substituting (11)-(14) into (10), overall noise power becomes

$$
\begin{aligned}
\overline{v_{\text {oai }}^{2}} & =\frac{k T \gamma}{G_{l} C_{t}}\left(g m_{2}+g m_{e x}+\frac{C_{t}}{g m_{1} L_{\text {pai }}}\right)= \\
& =\left.\frac{k T \gamma}{G_{l} C_{t}}\left(2 g m+g m_{e x}\right)\right|_{\substack{g m_{1}=g m_{2}=g m \\
C_{g}=C_{t}}}
\end{aligned}
$$

which corresponds with results published by Kaunisto [6].

For the degenerated gyrator-based resonator, Fig. 5. depicts the equivalent noise model.

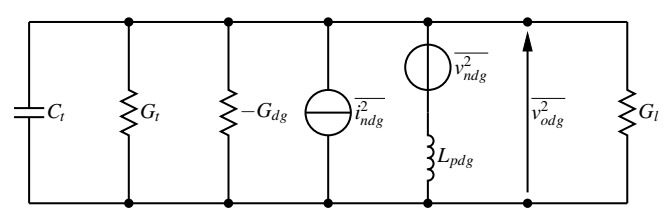

Fig. 5. Noise model of degenerated gyrator resonator.

The corresponding active inductor noise sources can be found analysing Fig. 6. and are equal to

$$
\begin{aligned}
& \overline{\overline{i_{n d g}^{2}}}=\overline{i_{d 2}^{2}}+\overline{v_{n R}^{2}} \frac{g m_{2}}{\Delta f} \frac{\omega^{2} C_{1}^{2} R_{f}^{2}+1}{\Delta f} \\
& \overline{\frac{v_{n d g}^{2}}{\Delta f}}=\frac{1}{g m_{1}^{2}}\left(\overline{\frac{i_{d 1}^{2}}{\Delta f}}+\frac{\overline{v_{n R}^{2}}}{\Delta f} \frac{\omega^{2} C_{1}^{2}}{\omega^{2} C_{1}^{2} R_{f}^{2}+1}\right)
\end{aligned}
$$

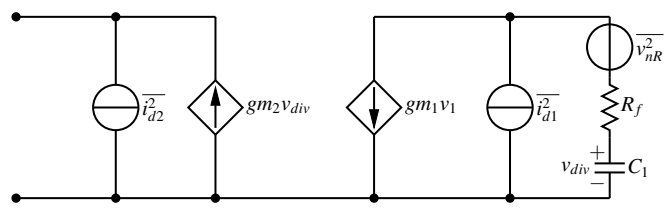

Fig. 6. Degenerated active inductor noise sources.

It can be seen that both generators are now correlated through $R_{f}$, hence to calculate the total resonator output noise, a transfer function related to this noise source is necessary. As before, both 
transconductors are represented by thermal channel noise, and have the same transfer functions as (11). The resistor noise transfer function is equal to:

$$
A_{n R d g}(j \omega)=\frac{1}{g m 1} \frac{1}{j \omega C_{1} R_{f}+1} \frac{j \omega\left(C_{1}+g m_{1} g m_{2} L_{p d g}\right)}{j \omega L_{p d g} G_{l}+1-\omega^{2} C_{t} L_{p d g}}
$$

Applying (16) and (17) into (10) the total output noise power can not be presented in a simplified analytical form and compared to the results given by (15). However, by a proper design of the degenerated gyrator, the $C_{1} R_{f}$ cut-off frequency may be smaller than the lower $3-\mathrm{dB}$ point of the tank thus minimising injected noise. The noise transfer function (17) becomes

$$
A_{n R d g}(j \omega) \approx \frac{1}{g m 1} \frac{1}{C_{1} R_{f}} \frac{C_{1}+g m_{1} g m_{2} L_{p d g}}{j \omega L_{p d g} G_{l}+1-\omega^{2} C_{t} L_{p d g}}
$$

and an approximated total output noise power using (10) is now

$$
\begin{aligned}
\overline{v_{o d g}^{2}} & \approx \frac{k T \gamma}{G_{l} C_{t}}\left[g m_{2}+\frac{C_{t}}{g m_{1} L_{p d g}}\left(1+\frac{\left(C_{1}+g m_{1} g m_{2} L_{p d g}\right)^{2}}{\gamma g m_{1} C_{1}^{2} R_{f}}\right)\right] \\
& =\frac{k T \gamma}{G_{l} C_{t}}\left[2 g m+\frac{1}{\gamma R_{f}}\left(1+\frac{C_{t}}{C_{1}}\right)^{2}\right] \mid \begin{array}{l}
g m_{1}=g m_{2}=g m \\
L_{p a i}=L_{p d g} \\
L p d g=C / g m^{2}
\end{array}
\end{aligned}
$$

The ratio of total noise power of the traditional active inductor (15) to that of the degenerated gyrator (19) can now be simplified. In the case of a low $\mathrm{Q}$ factor gyrators, it is possible to find $R_{f}$ and $C_{1}$ such

$$
\frac{1}{\gamma R_{f}}\left(1+\frac{C_{t}}{C_{1}}\right)^{2} \leq g m_{e x}
$$

giving an approximated total output noise ratio of

$$
\frac{\overline{v_{o a i}^{2}}}{\overline{v_{o d g}^{2}}} \geq 1
$$

Thus using this methodology, the theory shows that it is possible to obtain lower overall noise from a generic degenerated gyrator than from the traditional design. In practice, the freedom to choose different $R_{f}$ and $C_{1}$ combinations will allow optimal noise performance to be obtained, adjusting for the different levels of gyrator losses, resonant frequency and required power consumption.

\section{DYNAMIC RANGE}

In contrast to passive resonators, active filters suffer from limited dynamic range. The main factor limiting the dynamic range comes from the dependency of transistor parasitic elements on signal amplitude. As the induced inductance is proportional to the transconductance of both amplifiers, the resonant frequency of a tank is also signal dependent. Thus the upper limit of the gyrator-based resonator dynamic range may be defined as the maximum level for which a given frequency change is observed. There is no specified value for this parameter in literature, for the propose of this paper we define a frequency deviation equal to $1 \%$. The maximum signal level can be found either by applying a detailed Volterra analysis [8] or numerical simulation of the resonator. However, some indicative results may be found using a highly simplified analysis presented here.

If $\left|v_{\max A I}\right|$ represents the upper limit of the dynamic range and thus the maximum allowable signal at the transconductor input (see Fig. 1), then for a symmetrical gyrator-based resonator $\left(g m_{1}=g m_{2}=\right.$ $g m$ and $C_{g}=C_{t}$ ), this is also the maximum amplitude at the input of the feedback amplifier, $\left|v_{\text {ingm }_{2} A I}\right|$. If now $\left|v_{\max D G}\right|$ is defined the same way for the degenerated gyrator, then the maximum amplitude at the input of the second transconductor is now equal to

$$
\left|v_{\text {ingm }_{2} D G}\right|=\frac{g m_{1}}{\omega C_{p}} \frac{\left|v_{\max D G}\right|}{\sqrt{1+\left(\frac{\omega}{\omega_{H}}\right)^{2}}}
$$

If both resonator circuits use the same input and feedback transconductors respectively, then the voltages $\left|v_{\text {ingm } 2 A I}\right|$ and $\left|v_{\text {ingm } m_{2} D G}\right|$ should be equal, producing the same harmonic content in both gyrators. Analysing circuit from Fig. 3 and using (9) together with (22), the maximum input amplitude in comparison to the standard gyrator is now

$$
\frac{\left|v_{\max D G}\right|}{\left|v_{\max A I}\right|} \leq 1
$$

Therefore the degenerated gyrator has a lower upper limit of the dynamic range than the standard active inductor. This limitation is directly proportional to the amount of resonator losses that have to be compensated, yielding $-3 \mathrm{~dB}$ difference when both gyrators input losses are as high as gm. However, additional negative resistor influence on dynamic range has not been included. In addition, if the passively compensated resonator is designed for a lower noise value, the overall dynamic range may be still higher than in the standard approach.

\section{Simulation RESUlts}

To confirm the theoretical results from the previous sections, a comparative analysis of two resonators is presented. Both circuits have been designed to resonate at $434 \mathrm{MHz}$, within the lower European ISM band. This frequency range is attractive from circuit integration point of view as the passive resonator occupies significant chip area. As a test circuit, a simple grounded active inductor is used [9]. The equivalent small signal model reveals that input losses are approximately equal to the transconductance of a common source amplifier and the unloaded Q factor value is therefore very small. This particular circuit consumes a significant amount of static power and requires relatively large negative resistor for compensation, thus a suitable testbed for proposed method. The basic resonator structure

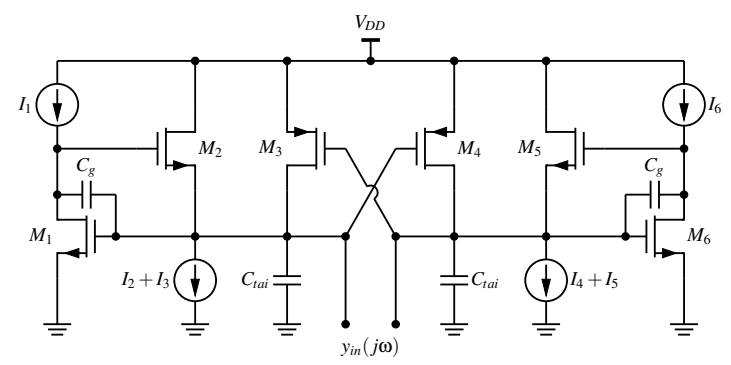

Fig. 7. Actively compensated active inductor resonator

is depicted in Fig. 7. The cross-coupled resistor is made of PMOS transistors, sized to fully utilize the voltage drop on the common drain transistors from the active inductor circuit and to generate less noise than its NMOS counterpart. All of the current sources are ideal to exclude its noise effecting the comparative analysis.

In the second case (see Fig. 8.) the same gyrator is used with the degeneration circuit. This active inductor, first published by Hsiao et al. [10] where highly-nonlinear capacitances were used, will be designed utilising the new methodology, (equations (4), (7) and (9)) which addresses the, until now, unresolved issues of noise, dynamic range and power consumption of passively compensated resonators. 


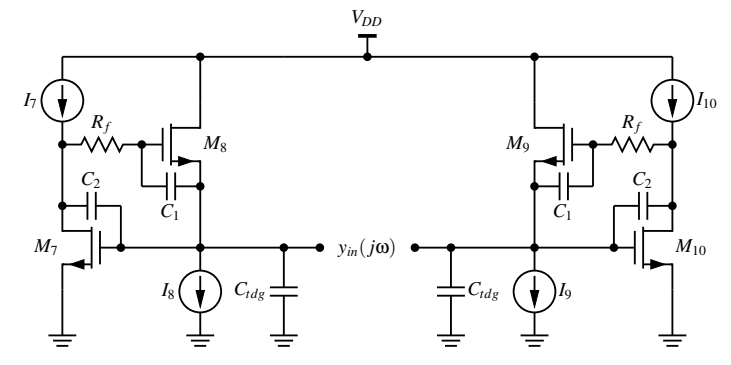

Fig. 8. Degenerated active inductor resonator.

Table I includes all necessary design parameters, for which circuits were simulated. In all devices, the gate length was set to $0.25 \mu \mathrm{m}$ to decrease thermal noise accordingly to UMC test noise analysis data. To ensure a proper resonator noise prediction, both resistors are modelled with thermal noise. Simulations were conducted in both

TABLE I

DEVICE SIZE AND CIRCUIT PARAMETERS

\begin{tabular}{|c|c|c|}
\hline \multicolumn{3}{|c|}{ Model: UMC MM180_REG18, L $=0.25 \mu \mathrm{m}$} \\
\hline \multirow{2}{*}{ Device } & \multirow{2}{*}{ Type } & $\mathrm{W}$ \\
\hline & & $\mu \mathrm{m} \times \mathrm{NF} \times \mathrm{M}$ \\
\hline $\mathrm{M}_{1,6,7,10}$ & \multirow{2}{*}{$\mathrm{N}$} & $5 \times 4 \times 1$ \\
\hline $\mathbf{M}_{2,5,8,9}$ & & $6 \times 6 \times 1$ \\
\hline $\mathrm{M}_{3,4}$ & $P$ & $4.2 \times 5 \times 2$ \\
\hline $\mathrm{V}_{\mathrm{DD}}$ & V & 1.8 \\
\hline $\mathrm{I}_{1,6,7,10}$ & \multirow{3}{*}{$\mathrm{mA}$} & 0.47 \\
\hline $\mathrm{I}_{2,5,8,9}$ & & 0.32 \\
\hline $\mathrm{I}_{3,4}$ & & 2.05 \\
\hline $\mathrm{C}_{\text {tai }}$ & \multirow{5}{*}{$\mathrm{pF}$} & 1.37 \\
\hline $\mathrm{C}_{\mathrm{g}}$ & & 1.45 \\
\hline $\mathrm{C}_{\mathrm{tdg}}$ & & 1.42 \\
\hline $\mathrm{C}_{1}$ & & $0.18^{*}$ \\
\hline $\mathrm{C}_{2}$ & & 0.4 \\
\hline $\mathrm{R}_{\mathrm{f}}$ & $\mathrm{k} \Omega$ & 2.8 \\
\hline \multicolumn{3}{|c|}{ *-for larger $M_{8,9}, C_{g s}$ can be used instead } \\
\hline
\end{tabular}

Eldo RF and Spectre, using UMC $0.18 \mu \mathrm{m}$ 1P6M process libraries for mixed mode design. Noise was simulated using AC analysis, then integrated over the frequency band from $400 \mathrm{MHz}$ to $500 \mathrm{MHz}$. Steady state large signal analysis was performed to find the input signal level for which the resonant frequency changes by $1 \%$, in this case $4.34 \mathrm{MHz}$. The presented active inductors experience harmonic expansion [8] i.e. the increased input signal amplitude causes an increase of inductance, hence shifting the resonant frequency down.

Table II presents the simulation results. Both circuits were simulated for unloaded $\mathrm{Q}$ and with a $5 \mathrm{k} \Omega$ load resistor connected between the differential input/output. It can be seen that the passively compensated circuit achieves both lower noise and larger maximum signal amplitude in comparison with the standard approach. In the case of the lower (loaded) Q, the total r.m.s. noise voltage is lower which corresponds to the results of Abidi [7]. Even though the $\mathrm{Q}$ values are slightly higher for the degenerated gyrator, proposed compensation method still generates around 1.5-3 dB less output noise than the traditional approach. The main advantage of the passive compensation can be seen in the significant decrease in power consumption, almost 3.6 times less current is necessary to bias the resonator. The dynamic range of both resonators is shown, revealing 3-5 dB gain in favor of the passive compensation, contrary to the highly simplified signal limit derivation included in the previous
TABLE II

SIMULATION RESULTS

\begin{tabular}{|c|c|c|c|c|c|}
\hline \multirow{2}{*}{\multicolumn{2}{|c|}{$\begin{array}{c}\begin{array}{c}\text { Circuit } \\
\text { type }\end{array} \\
\text { Simulator }\end{array}$}} & \multicolumn{2}{|c|}{$\begin{array}{l}\text { Actively compensated } \\
\text { resonator }\end{array}$} & \multicolumn{2}{|c|}{$\begin{array}{l}\text { Passively compensated } \\
\text { resonator }\end{array}$} \\
\hline & & Eldo & Spectre & Eldo & Spectre \\
\hline \multicolumn{2}{|c|}{$\mathrm{Q}_{0}\left(\mathrm{R}_{\mathrm{l}} \rightarrow \infty\right)$} & 22.6 & 23 & 31.4 & 31 \\
\hline $\mathrm{V}_{\mathrm{rms}}{ }^{*}$ & $\mu \mathrm{V}$ & 580 & 609 & 413 & 433 \\
\hline $\mathrm{V}_{\text {comp }}{ }^{* *}$ & $\mathrm{mV}$ & 15 & 13 & 20 & 16 \\
\hline DR & $\mathrm{dB}$ & 28.2 & 26.6 & 33.7 & 31.4 \\
\hline \multicolumn{2}{|c|}{$\mathrm{Q}_{\mathrm{l}}\left(\mathrm{R}_{\mathrm{l}}=5 \mathrm{k} \Omega\right)$} & 7.5 & 7.5 & 12.2 & 12 \\
\hline $\mathrm{V}_{\mathrm{rms}}{ }^{*}$ & $\mu \mathrm{V}$ & 290 & 305 & 244 & 249 \\
\hline $\mathrm{V}_{\text {comp }}{ }^{* *}$ & $\mathrm{mV}$ & 15 & 12 & 20 & 15 \\
\hline DR & $\mathrm{dB}$ & 34.3 & 31.9 & 38.3 & 35.6 \\
\hline $\mathrm{P}_{\mathrm{DC}}$ & $\mathrm{mW}$ & \multicolumn{2}{|c|}{10.22} & \multicolumn{2}{|c|}{2.84} \\
\hline
\end{tabular}

section. This indicates that a more detailed nonlinear analysis is necessary to fully describe large signal behavior of both circuits, especially in a presence of cross-coupled negative resistor.

\section{CONCLUSION}

In this paper we have presented a new design methodology for passive compensation of active inductor resonator losses. Enhancing the unwanted effect of parasitic RC components in active inductors, the proposed approach allows the design of sufficient negative resistance values inside a gyrator circuit. Comparative circuit simulation proved that a typical active inductor resonator performance is achieved with a significant reduction in power consumption, especially when the gyrator losses are high. This power can be conserved or utilised to further decrease resonator noise if necessary.

\section{ACKNOWLEDGMENTS}

Research presented in this paper was funded by a Centre for Telecommunications Value-Chain Research (SFI 03/CE3/I405) by Science Foundation Ireland under the National Development Plan. The authors gratefully acknowledge this support.

\section{REFERENCES}

[1] B. D. H. Tellegen, "The gyrator: a new electric network element," Phillips Research Reports, no. 3, pp. 81-101, 1948.

[2] B. Razavi, RF Microelectronics. Upper Saddle River, NJ, USA Prentice-Hall, Inc., 1998.

[3] J. Craninckx and M. Steyaert, "Low-noise voltage-controlled oscillators using enhanced LC-tanks," IEEE Transactions on Circuits and Systems II: Analog and Digital Signal Processing, vol. 42, no. 12, pp. 794-804, Dec. 1995.

[4] G. Szczepkowski and R. Farrell, "Negative resistance generation in degenerated gyrator," in Emerging Trends in Wireless Communications, 2008., Research Colloquim, Apr. 2008, pp. 59-62.

[5] Y. T. Wang and A. A. Abidi, "CMOS active filter design at very high frequencies," IEEE Journal of Solid-State Circuits, vol. 25, no. 6, pp. 1562-1574, Dec. 1990.

[6] R. Kaunisto, "Monolithic active resonator fitlers for high frequencies," Ph.D. dissertation, Helsinki Univerity of Technology, November 2000.

[7] A. A. Abidi, "Noise in active resonators and the available dynamic range," IEEE Transactions on Circuits and Systems I: Fundamental Theory and Applications, vol. 39, no. 4, pp. 296-299, Apr. 1992.

[8] P. Wambacq and W. M. Sansen, Distortion Analysis of Analog Integrated Circuits. Norwell, MA, USA: Kluwer Academic Publishers, 1998.

[9] M. Ismail, R. Wassenaar, and W. Morrison, "A high-speed continuoustime bandpass VHF filter in MOS technology," in Circuits and Systems, 1991., IEEE International Sympoisum on, June 1991, pp. 1761-1764.

[10] C. Hsiao, C. Kuo, C. Ho, and Y. Chan, "Improved quality-factor of 0.18-um CMOS active inductor by a feedback resistance design," IEEE Microwave and Wireless Components Letters, vol. 12, no. 12, pp. 467469, Dec. 2002. 